EMLyon Business School; OCE

\title{
AUDREY ROUZIÈS
}

IAE Toulouse ; CRM

\section{Ensemble mais différents}

\section{Ambivalence et mimétisme dans les dynamiques d'identification organisationnelle au sein des équipes composites}

Face aux transformations de l'emploi et du travail, des équipes composites regroupant à la fois des salariés et des travailleurs extérieurs deviennent une configuration productive de plus en plus répandue dans les entreprises. Or la performance de telles équipes nécessite un ciment qui n'est plus fourni par l'homogénéité des statuts d'emploi. L'identification organisationnelle constituant un facteur de performance, cet article contribue à la compréhension des antécédents spécifiques de l'identification organisationnelle pour les travailleurs dans des équipes composites. 
« éveloppons ensemble l'esprit d'équipe » tel est l'engagement de la Société générale envers ses collaborateurs. Mais de quelle équipe parle-t-on en réalité ? Si les salariés du groupe font effectivement l'objet de politiques de ressources humaines, qu'en est-il de leurs collègues de bureau qui sont considérés comme «externes»? En effet, les stratégies de flexibilité de la main-d'œuvre dans les entreprises et dans la banque en particulier ont conduit à la généralisation $\mathrm{du}$ recours à des travailleurs extérieurs (Everaere, 2012 ; Jacob, 2012). La relation d'emploi entre le travailleur et l'organisation revêt ainsi des formes diverses depuis le salarié "classique » à l'intérimaire en passant par le stagiaire et le prestataire. Même si les dirigeants des grands groupes continuent de s'adresser à «leurs salariés » et de mettre en œuvre des politiques sociales cherchant à développer un sentiment d'appartenance, la réalité sur le lieu de travail est tout autre. Souvent intégrés à des équipes de salariés, les travailleurs extérieurs partagent le quotidien d'une organisation qui n'est pas leur employeur. La littérature en management s'est déjà largement intéressée à l'identification des salariés présentant des statuts d'emploi identiques mais à notre connaissance aucune étude n'existe sur les processus d'identification dans des équipes dont les membres ont des statuts différents. Nous nous intéressons dans cet article aux modifications induites dans les dynamiques d'identification organisationnelle (Rousseau, 1998; George et Chattopadhyay, 2005; Ekmekci et Casey, 2011). Nous examinons ainsi les antécédents de l'identification des membres salariés et travailleurs extérieurs des équipes composites. Nous définissons dans un premier temps, un cadre d'analyse exploratoire issu de la littérature, puis nous présentons une étude de cas. Les résultats sont présentés puis discutés. Notre contribution porte sur l'étude des antécédents d'identification spécifiques dans le contexte d'une équipe dont les membres n'ont pas le même statut d'emploi.

\section{I - UN CADRE D'ANALYSE EXPLORATOIRE}

«L'observation des organisations en action débouche rapidement sur un étonnement majeur. Comment, en dépit des divergences d'intérêts des uns et des autres, d'un rapport lâche aux règles, d'un éclatement croissant des espaces de production, est-il encore possible de créer suffisamment de cohérence et de cohésion pour faire œuvre commune ?» (Lallement, 2007, p. 424). Cette citation du sociologue Michel Lallement nous ramène à la question fondamentale qui guide la problématique de l'article : comment des individus entretenant des relations avec l'organisation de natures différentes peuvent-ils encore s'identifier à elle ? Nous proposons de poser le cadre exploratoire en trois temps. Une première partie présente la tension entre des différences de statuts d'emploi et l'impératif de coopération dans l'entreprise. Une deuxième partie s'intéresse plus particulièrement aux antécédents de l'identification organisationnelle qui vont permettre d'améliorer ou non la coopération. Enfin, la troisième partie propose un cadre d'analyse des dynamiques d'identification dans les équipes composées à la fois de salariés et de travailleurs extérieurs. 


\section{Transformation de la relation d'emploi et coopération dans l'entreprise : la généralisation des équipes composites ?}

La généralisation du recours à des travailleurs extérieurs dans l'entreprise provient de la mise en œuvre de formes de flexibilité de l'emploi et du travail « amenant à une hétérogénéisation de la relation salariale, caractérisée notamment par une rupture dans la règle des trois unités : unité de lieu, unité de temps, unité d'action. » (BeaujolinBellet, 2004, p. 10). Des intérimaires, des consultants et prestataires, des stagiaires ou apprentis intègrent l'entreprise en qualité de travailleurs extérieurs. Cette situation peut créer des difficultés de coopération dues à la diversité des statuts d'emploi (Everaere, 2012) ainsi qu'à des sources de stress au travail supplémentaires (Beaujolin-Bellet et Schmidt, 2012). Selon cette approche, il est réputé difficile de coopérer avec des personnes dont le statut est différent. Or de plus en plus de salariés sont amenés à coopérer avec des travailleurs extérieurs (Jacob, 2012). Cette réalité contribue à la formation d'équipes composites que sont des équipes de travail «constituées par des salariés et des travailleurs externes partageant l'espace de travail de l'entreprise et des règles de fonctionnement d'équipe dans l'objectif d'atteindre un résultat défini de manière formelle par l'organisation ou par le groupe lui-même » (Jacob, 2012, p. 239). La coopération au sein de ces équipes particulières peut s'apparenter à de la coopération complémentaire (Dameron, 2005) fondée sur des différences de statuts d'emploi mais un intérêt commun de remplir les objectifs. Par ailleurs, la coopération communautaire (Dameron, 2005) est à l'œuvre dans les équipes composites dans la mesure où un travail identitaire se produit y compris dans les équipes aux affiliations organisationnelles diverses (Rousseau, 1998). Le processus d'enrôlement (Dameron, 2005) part du principe que les rôles se construisent dans l'interaction et que l'appartenance au groupe est renforcée par la division interne du travail attribuant un rôle effectif à chaque membre. Dans cette perspective, le sentiment d'appartenance devrait être également renforcé dans le processus d'enrôlement. Si Chédotel (2004) étudie de manière approfondie la relation entre l'identification et la coopération, comme elle le précise, la relation entre la coopération et l'identification mérite d'être étudiée en retour. Au final, la coopération complémentaire et communautaire contient des processus sociaux qui peuvent être compris comme des antécédents de l'identification au sens de la théorie de l'identité sociale.

\section{L'identification dans les équipes composites : quels antécédents ?}

Le concept d'identification sociale (Tajfel et Turner, 1979) s'est déployé depuis une trentaine d'années dans le champ du management (Albert et Whetten, 1985 ; Ashforth et Mael, 1989). La théorie de l'identité sociale (Abrams et Hogg, 1999, 1988 ; Hogg et Terry, 2001) et son prolongement la théorie de l'auto-catégorisation (Hogg et Terry, 2000; Tajfel et Turner, 1986) définissent l'identification sociale comme un lien entre un individu et un groupe. Ces théories soulignent trois hypothèses cœur dans le processus d'identification :

- L'identification est un processus par lequel un individu se définit - au moins en partie - par rapport à un groupe (hypothèse de catégorisation sociale). Elle implique l'inclusion de l'appartenance au groupe 
dans la définition de soi (self concept) de l'individu.

- Les individus, au travers d'un processus de comparaison sociale intergroupe, cherchent à clarifier les frontières entre leur endo-groupe (ingroup) et les exo-groupes (outgroups) en repérant les caractéristiques saillantes et distinctives de leur groupe d'appartenance.

Les individus, cherchent à s'identifier à des groupes perçus comme positifs afin de renforcer leur estime de soi.

La littérature antérieure sur l'identification organisationnelle s'est extensivement intéressée aux antécédents de l'identification (Dutton et al., 1994). Cependant, les travaux existants portent sur des groupes dans lesquels les individus ont des statuts d'emploi homogènes. Dans cet article, nous analysons les antécédents spécifiques à l'identification dans les équipes composites et contribuons ainsi à la littérature.

La caractéristique principale d'une équipe composite est de regrouper des individus dont le statut d'emploi est différent. Or, le statut est déterminant dans le processus d'identification. En effet, la théorie de l'identité sociale pose que l'individu s'identifie à un groupe pour lequel il perçoit une forte cohérence entre ses caractéristiques propres et les caractéristiques du groupe. De telle sorte que les membres identifiés à un même groupe se perçoivent identiques entre eux (endo-groupe/ingroup) et différents des autres groupes (exo-groupes/ outgroup). La généralisation du recours à des travailleurs extérieurs interroge donc la manière dont ces travailleurs s'identifient à l'organisation. Considérés comme "extérieurs » dans l'organisation dans laquelle ils travaillent et n'ayant pas forcément un autre employeur, la question de l'identi- fication organisationnelle se pose en des termes différents pour chaque statut au sein de l'équipe composite.

La littérature existante s'est déjà intéressée à l'équipe comme cible d'identification (Gundlach et al., 2006 ; Lembke et Wilson, 1998 ; Van Der Vegt et Bunderson, 2005). Au-delà de l'identification à l'équipe, les membres peuvent s'identifier à l'organisation. Dans cet article, nous nous intéressons à l'identification de membres d'une équipe composite à l'organisation et non pas à l'équipe. Plus spécifiquement, nous nous attachons à comprendre les antécédents de l'identification organisationnelle des membres d'une équipe composite.

George et Chattopadhyay (2005) montrent que les antécédents personnels de l'identification définis comme la qualité de l'interaction avec les membres permanents de l'organisation et la manière dont le travailleur est valorisé par l'organisation, vont être plus importants pour les prestataires dans leur identification à l'entreprise cliente que les antécédents impersonnels (comme le prestige, l'adéquation des valeurs et l'image positive perçue). Ekmekci et Casey (2011) montrent que plus les travailleurs temporaires interagissent avec les autres membres de l'organisation plus ils s'identifient à l'organisation au cours du temps. Ce résultat rejoint le résultat de George et Chattopadhyay (2005) sur la prépondérance des antécédents personnels de l'identification. Au final, les antécédents impersonnels de l'identification comme l'adéquation des valeurs entre le travailleur et l'organisation s'appliquent moins dans le cas des travailleurs extérieurs.

Partant des résultats obtenus dans les études sur les antécédents de l'identification, il apparaît que les salariés de l'entreprise vont 
plus s'identifier selon des antécédents impersonnels comme le prestige, l'adéquation des valeurs, et l'image positive perçue de l'organisation (Dutton et al., 1994 ; George et Chattopadhyay, 2005). Les prestataires et les travailleurs temporaires s'identifieront quant à eux plutôt selon des antécédents personnels comme la qualité des interactions entre collègues et avec la ligne managériale et la manière dont ils sont valorisés dans l'organisation (Ekmekci et Casey, 2011).

Pour mieux comprendre les dynamiques d'identification dans les organisations temporaires, Rousseau (1998) propose le concept d'identification située (situated identification). Cette forme élémentaire d'identification repose sur la perception d'un cadre de référence créé par des indices situationnels (situational cues) mettant en évidence les intérêts partagés et rendant saillante une identité. Les indices situationnels relèvent d'objets physiques comme une boîte à outils donnée aux opérateurs d'une usine ou des éléments plus abstraits comme une deadline commune ou une mission à accomplir. Rousseau (1998) précise que l'identification située est particulièrement visible dans les organisations du travail temporaires comme les campagnes politiques ou les équipes de tournage. L'identification située dans ces équipes est liée au fait que les individus réalisent une tâche spécifique dans un temps délimité, que cette tâche est partagée, importante et requière la collaboration entre des personnes détenant des expertises différentes (Rousseau, 1998, p. 221). Cette identification est temporaire car les indices situationnels qui la soutiennent disparaissent à la fin du projet. Dans une équipe de travail temporaire, les travailleurs relevant de statuts différents peuvent s'identifier au collectif le temps de la mission.

\section{Proposition de cadre d'analyse exploratoire dans le cas des équipes composites}

L'équipe composite est par nature temporaire car elle regroupe des salariés permanents et des travailleurs extérieurs employés de manière temporaire dans l'organisation. D'après Rousseau (1998), les membres de l'équipe composite sont susceptibles de développer une identification située en percevant des intérêts partagés et en ayant le sentiment de former un «nous » avec l'organisation. D'après George et Chattopahyay (2005) et Ekmekci et Casey (2011), les salariés s'identifient à l'organisation grâce à des antécédents impersonnels et les travailleurs extérieurs s'identifient à l'organisation grâce à des antécédents personnels. En résumé, les salariés membres d'une équipe composite présenteraient un processus d'identification organisationnelle

Figure 1 - Antécédents de l'identification organisationnelle

\begin{tabular}{|ll|}
\hline \multicolumn{1}{|c|}{ Type de travailleur } & \multicolumn{1}{c|}{ Spécificité des antécédents } \\
\hline Salariés & $\begin{array}{l}\text { Antécédents impersonnels (prestige, adéquation des valeurs, image } \\
\text { positive) } \\
\text { Travailleurs extérieurs }\end{array}$ \\
$\begin{array}{l}\text { Antécédents personnels (qualité des interactions entre collègues, avec la } \\
\text { ligne managériale) }\end{array}$ \\
\hline
\end{tabular}


combinant des antécédents impersonnels et des indices situationnels. Les travailleurs extérieurs membres d'une équipe composite présenteraient un processus d'identification combinant des antécédents personnels et des indices situationnels. Le cadre d'analyse exploratoire présenté ci-dessous (figure 2) s'inspire de la littérature existante qui, jusqu'à présent a analysé séparément les antécédents de l'identification organisationnelle des salariés et les antécédents de l'identification organisationnelle des externes. Nous combinons les travaux issus de cette littérature pour proposer un cadre d'analyse exploratoire sur les antécédents de l'identification des membres internes et externes d'une équipe composite.

Dans la suite de l'article, nous mettons ce cadre d'analyse à l'épreuve d'une étude de cas dont la méthodologie est décrite ciaprès.

\section{II - LE CAS GLOBAL DE L'ORGANISATION ET LES ÉQUIPES COMPOSITES}

\section{Contexte de l'entreprise sur la période étudiée}

L'entreprise Alpha est une grande entreprise française du secteur de l'énergie. Aux côtés des salariés, des travailleurs qualifiés « d'externes » réalisent des activités pour le compte d'Alpha avec des contrats de prestation, d'intérim, d'apprentissage etc. Cette catégorie d'externe marque une différence d'appartenance organisationnelle qui se traduit par une couleur de badge différente et une adresse de messagerie précisant la mention " externe » dans le libellé. Cependant, dans les bureaux d'Alpha, les salariés et les externes partagent les mêmes espaces et leurs noms sont précisés à l'entrée des bureaux sans que les qualités de salarié ou d'externe ne soient notées. Il subsiste ainsi une certaine ambiguïté entre les statuts de salarié et d'externe lorsque l'on observe les individus au travail. Pour la direction des services généraux, la répartition est équilibrée, moitié salariés et moitié externes. Cela s'explique par la forte externalisation des activités de services généraux comme la maintenance des équipements, le nettoyage, l'accueil ou la restauration. La direction des services généraux, largement considérée comme périphérique par rapport au cœur de métier de l'entreprise, ne fait pas l'objet d'une politique de recrutement prioritaire. Les managers ont donc recours à l'intérim ou à des contrats d'apprentissage en cas de besoin.

Figure 2 - Proposition de cadre d'analyse exploratoire

\begin{tabular}{|lc|}
\hline \multicolumn{1}{|c|}{ Type de travailleur } & \multicolumn{1}{c|}{ Spécificité des antécédents } \\
\hline $\begin{array}{l}\text { Travailleurs dans une } \\
\text { équipe composite } \\
- \text { dont membres salariés } \\
-\begin{array}{l}\text { dont membres travailleurs } \\
\text { extérieurs }\end{array}\end{array}$ & Indices situationnels (deadline commune, outils partagés) \\
\hline
\end{tabular}




\section{MÉTHODOLOGIE}

Le choix d'une démarche qualitative abductive fondée sur de l'observation participante

Nous tirons nos analyses d'observations récoltées lors d'une enquête de terrain menée dans l'entreprise Alpha au sein de la direction des services généraux dans le cadre d'une thèse en convention industrielle de formation par la recherche (CIFRE). La méthode d'observationparticipante a été mise en œuvre d'avril 2009 à septembre 2011 par l'immersion à plein temps de l'un des auteurs.

\section{Le choix d'une étude de cas enchâssés pour rendre compte de la dynamique}

L'un des auteurs a participé de manière indirecte aux équipes étudiées et a pu récolter le matériau suivant :

- Notes de terrain dans journal sous format word : 3 fichiers word soit 7 pages exploitées pour l'article sur 369 fichiers enregistrés sur le terrain.

- Comptes rendus de réunions : 2 comptes rendus exploités/des centaines enregistrés.

- Documents de travail : 1 annuaire exploité/3 enregistrés, 1 planning des congés exploité/3 enregistrés, 1 tableau de suivi/des centaines

- Documents internes : 1 newsletter exploitée/5 parues, lettres de mission signée par la directrice, 1 contrat de prestation et 1 cahier des charges, 2 organigrammes, 2 e-mails/des centaines archivés, 1 entretien annuel/des dizaines archivés.

- Entretiens informels : 3 notes d'humeurs exploitées/des dizaines rédigées après une conversation informelle pendant l'immersion sur le terrain.

Les notes d'humeur (Hocquelet, 2013) permettent en particulier de repérer des éléments saillants du terrain qui suscitent l'étonnement du chercheur. L'objectif étant d'étudier une dynamique, la méthode des cas enchâssés (Musca, 2006) est pertinente pour rendre compte des dynamiques d'identification dans chaque équipe sans perdre de vue le cas global de l'organisation.

Le premier auteur apporte les données et le second auteur apporte le cadre théorique. La spécification des cas s'élabore au travers d'une discussion entre les deux auteurs. Le risque de circularité (Dumez, 2013) est ainsi maîtrisé grâce à une double interprétation des données, l'une selon les éléments du terrain (perspective emic, Garsten, 2011) et l'autre selon une théorie (perspective etic, Garsten, 2011).

La direction générale d'Alpha lance en 2009 un projet d'aménagement d'un nouveau site pour regrouper les salariés de la région parisienne, passant ainsi d'une vingtaine de bâtiments répartis sur la région parisienne à cinq sites. Cette évolution conduit la directrice à mettre en place une nouvelle structure organisationnelle plus adaptée. Pour ce faire, elle s'appuie sur une équipe composite qui porte le nom du projet: Delta T1. Les membres de cette équipe sont des salariés rattachés à la direction des services généraux et des externes. Lors de la mise en place de la nouvelle 
structure, les membres salariés et externes de Delta T1, qui étaient missionnés sur des activités techniques, sont regroupés au sein d'un pôle intitulé " Pôle multitechnique ». Nous étudions ces deux équipes composites - Delta T1 et Pôle multitechnique - sous la forme de deux cas enchâssés dans le cas de l'entreprise Alpha.

\section{Cas 1 : l'équipe-projet Delta T1 (décembre 2009-juin 2010)}

L'équipe Delta T1 a pour mission de piloter la mise en exploitation des prestations de services généraux du nouveau siège d'Alpha. Ce projet est central à la fois pour la réorganisation de la direction et pour assurer la pérennité des activités de

Tableau 1 - Les différents périmètres de l'équipe

\begin{tabular}{|c|c|c|c|c|c|c|}
\hline Personnes & $\begin{array}{c}\text { Statut } \\
\text { d'emploi }\end{array}$ & Fonction & $\begin{array}{c}\text { Équipe } \\
\text { initiale } \\
\text { (déc. 2009) }\end{array}$ & $\begin{array}{c}\text { Équipe } \\
\text { d'après } \\
\text { l'annuaire } \\
\text { du projet } \\
\text { (mars 2010) }\end{array}$ & $\begin{array}{c}\text { Équipe } \\
\text { d'après } \\
\text { planning } \\
\text { des congés } \\
\text { (avril 2010) }\end{array}$ & $\begin{array}{c}\text { Photo } \\
\text { newsletter } \\
\text { (mai 2010) }\end{array}$ \\
\hline Josette & Salariée & Services & & $\checkmark$ & $\checkmark$ & \\
\hline Didier & Salarié & Plans & $\checkmark$ & $\checkmark$ & $\checkmark$ & $\checkmark$ \\
\hline Jean-Claude & Salarié & Sécurité & & & $\checkmark$ & $\checkmark$ \\
\hline Bruno & Salarié & Technique & $\checkmark$ & & $\checkmark$ & $\checkmark$ \\
\hline Yann & Salarié & Audio & $\checkmark$ & & $\checkmark$ & $\checkmark$ \\
\hline Céline & Prestataire & Services & $\checkmark$ & $\checkmark$ & $\checkmark$ & $\checkmark$ \\
\hline Gilles & Salarié & Chef projet & $\checkmark$ & $\checkmark$ & $\checkmark$ & $\checkmark$ \\
\hline Thierry & Salarié & Services & & & $\checkmark$ & \\
\hline Lydie & Salarié & Assistante & $\checkmark$ & $\checkmark$ & $\checkmark$ & \\
\hline Rémi & Prestataire & Appui-chef & $\checkmark$ & $\checkmark$ & $\checkmark$ & \\
\hline Jean-Luc & Salarié & Technique & & & $\checkmark$ & $\checkmark$ \\
\hline Paula & Salarié & Services & & $\checkmark$ & $\checkmark$ & \\
\hline Fabien & Salarié & Services & & & $\checkmark$ & \\
\hline Raymond & Salarié & Mobilier & $\checkmark$ & $\checkmark$ & $\checkmark$ & $\checkmark$ \\
\hline Frédéric & Salarié & Sécurité & & $\checkmark$ & $\checkmark$ & $\checkmark$ \\
\hline Sarah & Intérimaire & Sécurité & & & $\checkmark$ & $\checkmark$ \\
\hline Luc & Salarié & Sécurité & & $\checkmark$ & $\checkmark$ & $\checkmark$ \\
\hline
\end{tabular}


services généraux au sein de l'entreprise. Initialement, l'équipe est composée de six salariés détachés de leur poste à la direction des services généraux et de deux prestataires déjà en mission dans l'entreprise. À cette équipe initiale constituée de salariés et de prestataires s'ajoutent, en fonction des besoins, d'autres salariés ou d'autres « externes ». Ainsi, le périmètre de l'équipe évolue de décembre 2009 à mai 2010 comme le montre le tableau 1 à partir de quatre documents attestant de la composition de l'équipe à un moment donné.

$\mathrm{Au}$ final, l'équipe est passée de huit membres dont deux prestataires à dix-sept membres dont deux prestataires et une intérimaire. Ces trois travailleurs extérieurs étaient déjà en mission dans l'entreprise et ont été sélectionnés pour leurs compétences et leur connaissance de l'entreprise. Au sein de l'équipe Delta T1 se forme une sous- équipe technique qui devient l'équipe Pôle multitechnique dans la nouvelle structure de la direction des services généraux.

\section{Cas 2 : l'équipe Pôle multitechnique (juin 2010-septembre 2011)}

À partir du mois de juin 2010, la nouvelle structure de la direction des services généraux est officiellement mise en place et annonce la fin du mode projet. La souséquipe du projet Delta T1 devient une entité officielle de la direction des services généraux appelée Pôle multitechnique. L'équipe du Pôle multitechnique est composée de cinq salariés dans sa représentation officielle, de quatre salariés et de trois prestataires dans l'organigramme opérationnel.

$\mathrm{Au}$ sein de ces deux équipes composites, nous proposons d'analyser les situations individuelles présentant des éléments critiques dans le processus d'identification du travailleur. Le tableau 2 présente les

Tableau 2 - Présentation des personnes dans les deux équipes composites étudiées

\begin{tabular}{|c|c|c|}
\hline & $\begin{array}{c}\text { Cas } 1 \\
\text { Équipe Delta T1 }\end{array}$ & $\begin{array}{l}\text { Cas 2 } \\
\text { Équipe Pôle multitechnique }\end{array}$ \\
\hline $\begin{array}{l}\text { Travailleurs } \\
\text { prestataires }\end{array}$ & $\begin{array}{l}\text { Céline } \\
\text { Incident observé lors du processus de } \\
\text { changement organisationnel }\end{array}$ & $\begin{array}{l}\text { Cédric } \\
\text { Incidents montrant l'attachement à } \\
\text { Alpha }\end{array}$ \\
\hline \multirow[t]{2}{*}{ Salariés } & $\begin{array}{l}\text { Jean-Luc } \\
\text { S'investit dans l'équipe jusqu'à en } \\
\text { devenir un membre à part entière }\end{array}$ & $\begin{array}{l}\text { Jean-Luc } \\
\text { Obtient des rétributions financières } \\
\text { pour son implication dans le projet } \\
\text { Delta } \mathrm{T} 1 \text { mais perd la responsabilité } \\
\text { officielle de l'équipe qu'il manageait } \\
\text { dans l'ancienne structure }\end{array}$ \\
\hline & $\begin{array}{l}\text { Josette } \\
\text { Fait part de son regret de la qualité des } \\
\text { relations interpersonnelles après la fin } \\
\text { du projet. }\end{array}$ & \\
\hline
\end{tabular}


individus choisis en fonction des statuts d'emploi de prestataire et de salarié et de l'appartenance à une des équipes étudiées.

\section{III - RÉSULTATS EXPLORATOIRES}

Nous présentons dans un premier temps les constats du terrain relatifs au choix des cibles d'identification (tableau 3). Puis les tableaux 4 et 5 donnent à voir les situations individuelles prises dans chaque cas étudié pour montrer la manière dont les antécédents personnels se manifestent. Dans un troisième temps, nous présentons les analyses éclairant les antécédents impersonnels et enfin nous présentons le rôle ambivalent des indices situationnels (tableau 6).

Le tableau 3 montre que le référent identitaire des membres d'une équipe composite, qu'ils soient salariés ou externes, est bien cette équipe. En effet, pris séparément, les individus se définissent comme « internes » ou « externes »; mais lorsqu'ils sont dans un contexte qui rend saillante l'équipe, tous les individus membres se catégorisent en « Delta T1 ». L'appartenance à une équipe gomme les frontières entre internes et externes.

Le tableau 4 présente l'intégration de chacun des individus dans l'équipe composite et les évènements illustrant un processus d'identification reposant sur des antécédents personnels.

Concernant les antécédents impersonnels et la dimension de prestige en particulier, un fondement impersonnel important dans la littérature sur l'identification, nous avons pu noter que l'équipe projet Delta T1 était reconnue par tous les travailleurs et la hiérarchie de la direction. Pendant la période d'observation, l'équipe Delta T1 a fait l'objet d'un numéro spécial dans la newsletter de la direction des services généraux. On y voit Rémi, le prestataire, se faire interviewer au nom de l'équipe ainsi qu'une photo rassemblant les membres salariés et externes de l'équipe. Cet article dans la newsletter traduit la reconnaissance de l'équipe par la hiérarchie de la direction des services généraux. En effet, toutes les équipes composites présentes au sein de cette même direction ne sont pas mises en valeur de la sorte dans un support de communication interne. Ces éléments ne nous permettent pas de voir directement un lien entre l'antécédent et l'identification, en revanche, cela démontre l'intérêt de prendre en compte les antécédents impersonnels liés au prestige pour mesurer l'identification des travailleurs extérieurs. Au final, ces derniers sont exposés aux mêmes mécanismes

Tableau 3 - Verbatims récoltés sur le terrain selon le statut d'emploi

\begin{tabular}{|c|c|c|}
\hline & Salariés & Travailleurs extérieurs \\
\hline $\begin{array}{l}\text { Identification } \\
\text { à l'organisation }\end{array}$ & $\begin{array}{l}\text { « je suis interne } » \\
\text { « je suis Alpha }\end{array}$ & $\begin{array}{c}\text { « je suis externe » vient dans un premier temps } \\
\text { et parfois on entend préciser « je suis prestataire » } \\
\text { ou « je suis stagiaire » }\end{array}$ \\
\hline $\begin{array}{l}\text { Identification } \\
\text { à l'équipe }\end{array}$ & « je suis Delta T1» & « je suis Delta T1» \\
\hline
\end{tabular}


Tableau 4 - Synthèse des situations individuelles permettant de mettre en avant les antécédents personnels

\begin{tabular}{|c|c|c|c|}
\hline \multirow[b]{2}{*}{$\begin{array}{l}\text { Céline } \\
\text { Prestataire } \\
\text { employée } \\
\text { par la société } \\
\text { JFK }\end{array}$} & \multirow{2}{*}{$\begin{array}{l}\begin{array}{c}\text { Mode d'entrée dans } \\
\text { l'équipe composite }\end{array} \\
\mathbf{2 0 0 9} \\
\text { En mission pour une } \\
\text { autre équipe projet } \\
\mathbf{2 0 1 0} \\
\text { le chef de projet } \\
\text { Delta T1 demande } \\
\text { qu'elle rejoigne son } \\
\text { équipe, la directrice } \\
\text { négocie avec la } \\
\text { direction des achats }\end{array}$} & \multicolumn{2}{|c|}{ Évènements liés à un processus d'identification } \\
\hline & & $\begin{array}{l}\text { Avril } \mathbf{2 0 1 0} \\
\text { Céline fait remonter par } \\
\text { Luc ses inquiétudes, } \\
\text { le chef de projet ne } \\
\text { l'informe pas des consé- } \\
\text { quences de la réorganisa- } \\
\text { tion sur sa mission }\end{array}$ & $\begin{array}{l}\text { Mai } 2010 \text { en réunion de pilotage } \\
\text { "Quid des missions de Céline: } \\
\text { Redéfinir ses missions pour les } \\
6 \text { mois qui restent. Bien cadrer } \\
\text { ses missions. Rentabiliser sa com- } \\
\text { pétence. Rencontrer sa respon- } \\
\text { sable et optimiser ses missions. » } \\
\text { Au final, elle poursuit sur une } \\
\text { mission de gestion des transferts } \\
\text { de bureaux }\end{array}$ \\
\hline $\begin{array}{l}\text { Cédric } \\
\text { Prestataire } \\
\text { employé par } \\
\text { la société } \\
\text { JFK }\end{array}$ & $\begin{array}{l}\mathbf{2 0 1 0} \\
\text { Intègre l'équipe Pôle } \\
\text { multitechnique et } \\
\text { reprend certaines } \\
\text { activités de Céline }\end{array}$ & $\begin{array}{l}\text { Février } 2011 \\
\text { Cédric sait que son } \\
\text { entreprise touche trois } \\
\text { fois plus de Alpha que } \\
\text { lui ce qu'il touche en } \\
\text { salaire de son entreprise. } \\
\text { Pourtant il est impliqué } \\
\text { et motivé pour Alpha. } \\
\text { Il dit qu'il a beaucoup } \\
\text { de choses à apprendre } \\
\text { et que l'expérience chez } \\
\text { Alpha lui permet d'avoir } \\
\text { de l'autonomie. (notes } \\
\text { terrain) }\end{array}$ & $\begin{array}{l}\text { Mars 2010 } \\
\text { Signature mail de Cédric : } \\
\text { Cédric XXXXX - } 0144222101 \\
-0623443404 \\
\text { cedric.XXXXX@externe. } \\
\text { gammasigma.com } \\
\text { T1 - } 20^{\mathrm{e}} \text { étage - B. 20-073-B } \\
\text { En prestation de AMO } \\
\text { de services généraux (JFK) } \\
\text { Direction des services généraux } \\
\text { 1 place X } \\
\text { 92930 La Défense Cedex } \\
\text { Alpha } \\
\text { Cédric utilise le modèle de } \\
\text { signature fourni par Alpha mais } \\
\text { précise discrètement qu'il est en } \\
\text { prestation pour le compte de JFK }\end{array}$ \\
\hline $\begin{array}{l}\text { Jean-Luc } \\
\text { Salarié } \\
\text { cadre, rejoint } \\
\text { la direction } \\
\text { des services } \\
\text { généraux en } \\
2009\end{array}$ & $\begin{array}{l}2009 \\
\text { Rejoint le projet } \\
\text { Delta T1 d'abord } \\
\text { comme interlocuteur } \\
\text { puis comme membre } \\
\text { à part entière }\end{array}$ & $\begin{array}{l}\text { Novembre } \mathbf{2 0 1 0} \\
\text { Lors de l'entretien } \\
\text { annuel : «Les missions } \\
\text { confiées sont intéressantes } \\
\text { et les connaissances } \\
\text { acquises m'ont permis } \\
\text { d'évoluer sereinement } \\
\text { chez Alpha. Je remercie } \\
\text { ma hiérarchie actuelle } \\
\text { pour la confiance qu'elle } \\
\text { me témoigne ainsi que } \\
\text { pour les perspectives } \\
\text { évoquées » }\end{array}$ & $\begin{array}{l}\text { Mars } 2011 \\
\text { Déçu par sa hiérarchie qui ne l'a } \\
\text { pas consulté sur son changement } \\
\text { de bureau alors que ce sont les } \\
\text { collègues de son équipe qui l'ont } \\
\text { informé } \\
\text { Mai } 2011 \\
\text { Il ne change pas de bureau et } \\
\text { finit par obtenir un rôle officiel } \\
\text { de manager, mais sa demande } \\
\text { de MBA pour devenir cadre } \\
\text { dirigeant n'est pas acceptée }\end{array}$ \\
\hline $\begin{array}{l}\text { Josette } \\
\text { Salariée } \\
\text { cadre, rejoint } \\
\text { la direction } \\
\text { des services } \\
\text { généraux en } \\
2009\end{array}$ & $\begin{array}{l}\mathbf{2 0 0 9} \\
\text { Intègre l'équipe } \\
\text { Delta T1 sur le projet } \\
\text { de déploiement d'un } \\
\text { outil informatique } \\
\text { spécifique pour la } \\
\text { gestion des services } \\
\text { aux occupants des } \\
\text { bureaux }\end{array}$ & $\begin{array}{l}2010 \\
\text { "Avec Delta T1, on était } \\
\text { solidaires sur les tâches } \\
\text { à réaliser, on prenait les } \\
\text { pauses cafés et cigarettes } \\
\text { ensemble, c'était convi- } \\
\text { vial même si le boulot } \\
\text { était dur. » (notes terrain) }\end{array}$ & $\begin{array}{l}2011 \\
\text { "Maintenant que le projet est } \\
\text { terminé, c'est chacun pour soi. » } \\
\text { (notes terrain) }\end{array}$ \\
\hline
\end{tabular}


Tableau 5 - Synthèse des résultats sur les antécédents personnels

\begin{tabular}{|l|l|l|}
\hline \multicolumn{1}{|c|}{$\begin{array}{c}\text { Antécédents } \\
\text { personnels }\end{array}$} & \multicolumn{1}{|c|}{ Salariés } & \multicolumn{1}{c|}{ Prestataires } \\
\hline $\begin{array}{l}\text { Qualité des } \\
\text { relations entre } \\
\text { collègues }\end{array}$ & $\begin{array}{l}\text { Josette regrette la configuration de } \\
\text { l'équipe Delta T1 où elle trouvait que } \\
\text { les relations étaient particulièrement } \\
\text { bonnes. }\end{array}$ & $\begin{array}{l}\text { Céline parle de ses inquiétudes à } \\
\text { Luc, un salarié de l'équipe Delta T1 } \\
\text { qui remonte cette information à la } \\
\text { directrice. }\end{array}$ \\
\hline $\begin{array}{l}\text { Appréciation } \\
\text { de la hiérarchie }\end{array}$ & $\begin{array}{l}\text { Jean-Luc est déçu par sa hiérarchie } \\
\text { lorsqu'elle ne fait pas l'effort de lui } \\
\text { dire qu'il allait changer de bureau. }\end{array}$ & $\begin{array}{l}\text { Cédric est membre de l'équipe } \\
\text { de Jean-Luc comme l'indique } \\
\text { l'organigramme opérationnel. Mais } \\
\text { de son côté, il signe ses e-mails } \\
\text { en reprenant le modèle donné } \\
\text { aux salariés d'Alpha, en précisant } \\
\text { néanmoins en petits caractères son } \\
\text { statut de prestataire. }\end{array}$ \\
\hline
\end{tabular}

Tableau 6 - Synthèse de l'ambivalence des indices situationnels

\begin{tabular}{|c|c|c|}
\hline Indices situationnels & Marque la distinction & Renforce la similitude \\
\hline $\begin{array}{l}\text { Port obligatoire du badge } \\
\text { d'accès aux bureaux de } \\
\text { l'entreprise }\end{array}$ & $\begin{array}{l}\text { Les salariés ont un badge vert } \\
\text { et les externes un badge gris. }\end{array}$ & Tout le monde porte un badge. \\
\hline $\begin{array}{l}\text { Organisation d'évènements } \\
\text { pour les travailleurs de la } \\
\text { direction }\end{array}$ & $\begin{array}{l}\text { Les salariés sont invités en } \\
\text { priorité et les externes sont } \\
\text { invités « en fonction de la } \\
\text { place disponible » (extrait } \\
\text { d'un mail du directeur). }\end{array}$ & $\begin{array}{l}\text { Lors de l'évènement, des } \\
\text { externes sont mêlés aux } \\
\text { salariés et cela paraît normal. }\end{array}$ \\
\hline Activités quotidiennes & & $\begin{array}{l}\text { Postes de travail côte à côte, } \\
\text { même expérience du travail à } \\
\text { réaliser, deadlines communes. }\end{array}$ \\
\hline $\begin{array}{l}\text { Documents internes } \\
\text { d'organisation }\end{array}$ & $\begin{array}{l}\text { L'annuaire de l'équipe projet } \\
\text { Delta } \mathrm{T} 1 \text { précise à la fois } \\
\text { l'employeur et l'appartenance } \\
\text { à Delta T1. }\end{array}$ & $\begin{array}{l}\text { Des organigrammes indiquent } \\
\text { les externes et leurs rôles. }\end{array}$ \\
\hline
\end{tabular}

de reconnaissance que les salariés lorsqu'ils travaillent dans des équipes composites. Concernant les indices situationnels, le tableau 6 illustre leur ambivalence sur le lieu de travail.
Nous avons pu observer, dans l'entreprise Alpha des indices situationnels favorisant la construction d'une identité commune aux membres externes et internes de l'équipe composite. Cependant selon le 
type d'indice situationnel observé, certains renforcent la distinction (distinctiveness) entre les externes et les internes alors que d'autres renforcent la similitude (sameness) entre les employés de statuts différents. L'ambivalence des indices est de nature à renforcer l'identification du travailleur au groupe par les processus de travail tout en relativisant son appartenance au travers de procédures administratives distinctes. En somme, pour des individus aux statuts différents, l'appartenance à l'équipe engendre des processus d'identification mimétiques intra-groupe. Hors d'une équipe composite, les distinctions intergroupes entre salariés et travailleurs extérieurs perdurent, ce qui rejoint les résultats de George et Chattopadhyay (2005).

\section{IV - DISCUSSION ET CONCLUSION}

Contrairement à ce que l'on aurait pu penser au regard de la littérature sur laquelle se fonde cet article, les salariés membres d'une équipe composite expriment l'importance d'antécédents personnels comme la qualité des relations entre collègues et avec la ligne hiérarchique dans leur discours sur l'organisation. Pour les travailleurs extérieurs membres d'une équipe composite, il apparaît que la reconnaissance de l'organisation envers l'équipe les inclut et donc les expose à des antécédents plus impersonnels comme le prestige du groupe d'appartenance. En effet, les membres de l'équipe partagent au quotidien les mêmes conditions de travail. Les externes sont exposés aux mêmes contraintes mais également aux mêmes mécanismes de reconnaissance que les internes. Cette exposition aux mêmes conditions de travail conduit à un processus mimétique. Contrairement aux travaux antérieurs (George et Chattopadhyay, 2005) qui séparaient les antécédents de l'identification en fonction du statut des travailleurs, nos résultats exploratoires conduisent à observer une convergence des antécédents de l'identification suivant un processus mimétique entre les membres d'une équipe composite.

Ces résultats en désaccord avec la littérature existante s'expliquent par le fait que les travaux précédents analysaient séparément les antécédents de l'identification organisationnelle de salariés et les antécédents de l'identification organisationnelle des externes; alors que nous analysons conjointement les antécédents de l'identification organisationnelle au sein d'équipes composites regroupant les différents types de statuts. Il s'agit d'un résultat important car, en prenant en compte la dimension composite de l'équipe, on observe que les mécanismes d'identification déjà balisés dans la littérature sur les équipes dont les membres ont un statut d'emploi homogène s'appliquent différemment. Cela conduit à amender les résultats existants dans la littérature et souligne l'intérêt d'un management spécifique des processus d'identification dans ces équipes composites.

Ensuite, le caractère ambivalent de tous les antécédents et indices étudiés est un résultat important. Les salariés ont des comportements ambivalents qui se traduisent dans les interactions avec les travailleurs extérieurs, de même pour la hiérarchie, comme en témoigne la reconnaissance donnée à une équipe composite dans la communication interne. Ainsi, l'organisation reconnaît à certains moments la participation des travailleurs extérieurs tout en les excluant à d'autres. Les salariés se sentent à la fois épaulés et envahis par ces 
externes qui constituent un soutien offert par l'organisation mais également une menace pour leurs postes. Ces résultats complètent le travail de Rousseau (1998) qui ne prenait en compte dans son analyse que les effets facilitateurs des indices situationnels. Tout l'enjeu est de comprendre comment cette ambivalence des antécédents va influer sur l'identification des salariés et des travailleurs extérieurs.

Suite à cette étude exploratoire, il serait intéressant d'approfondir ces premiers résultats en raffinant l'analyse grâce à une approche longitudinale qui permettrait de comprendre si les antécédents changent au fil du temps pour chacun des types d'acteur (interne vs externe), et notamment quels rôles jouent l'ambivalence et le mimétisme intra-groupe au sein de l'équipe composite dans cette évolution. De même, des études quantitatives futures permettraient de compléter l'approche exploratoire exposée ici afin d'évaluer précisément les différents niveaux d'identification des salariés et des travailleurs extérieurs pour chacune des cibles repérées.

Cet article permet d'éclairer les managers sur les dynamiques d'identification à l'œuvre dans les nouvelles configurations productives de l'entreprise telles que les équipes composites. Les dirigeants d'unités comme la direction des services généraux étudiée ici, ont une vision des individus qui sont salariés et ceux qui sont externes. Quant aux managers en charge d'animer des équipes composites, ils font face à des difficultés pratiques pour concilier un management d'équipe avec la gestion quotidienne de travailleurs aux statuts différents. À chacun de ces niveaux hiérarchiques, une meilleure compréhension des processus identitaires à l'œuvre dans ces équipes éclaire sous un angle nouveau les problèmes de coopération et les tensions des individus au travail. Il s'agit d'ajuster ici les représentations des managers pour développer des pratiques qui actent que l'entreprise n'est plus composée uniquement de salariés.

\section{BIBLIOGRAPHIE}

Abrams D., Ando K. et Hinkle S. (1998). "Psychological attachment to the group: crosscultural differences in organizational identification and subjective norms as predictors of workers' turnover intentions", Personality and Social Psychology Bulletin, vol. 24, $\mathrm{n}^{\circ} 10$, p. 1027-1039.

Albert S. et Whetten D.A. (1985). "Organizational identity", Research in Organizational Behavior, vol. 7, p. 263-295.

Ashforth B.E. et Mael F. (1989). "Social identity theory and the organization", Academy of Management Review, vol. 14, n 1, p. 20-39.

Beaujolin-Bellet R. et Schmidt G. (2012). « Gestion des ressources humaines, du travail et de l'emploi. Quelles "bonnes théories" pour infléchir les "mauvaises pratiques" ? », Revue française de gestion, vol. 38, n 228-229, p. 41-57. 
Beaujolin-Bellet R. (2004). «Introduction. Aux sources des flexibilités : quelles transformations? », Flexibilités et performances, R. Beaujolin-Bellet, La Découverte « Recherches », p. 9-19.

Chédotel F. (2004). «Avoir le sentiment de faire partie d'une équipe : de l'identification à la coopération»,M@n@gement, vol.7,n³,p.161-193.

Dameron S. (2005). « La dualité du travail coopératif », Revue française de gestion, vol. 31, $\mathrm{n}^{\circ} 158$, p. $105-120$.

Dumez H. (2013). Méthodologie de la recherche qualitative, Vuibert.

Dutton J.E., Dukerich J.M. et Harquail C.V. (1994). “Organizational images and members identification”, Administrative Science Quarterly, vol. 39, n² 2, p. 239-263.

Ekmekci O. et Casey A. (2011). "Computer simulation exploring organizational identification for contingent workers”, Team Performance Management, vol. 17, n 5/6, p. 279-298.

Everaere C. (2012). «Flexibilité appliquée aux ressources humaines », Revue française de gestion, vol. $38, \mathrm{n}^{\circ} 221$, p. 13-32.

Garsten C. (2011). «Recherches qualitative dans les organisations: éléments méthodologiques », Le Libellio d'Aegis, vol. 7, n 2, p. 3-13.

George E. et Chattopadhyay P. (2005). "One foot in each camp: the dual identification of contract workers”, Administrative Science Quarterly, vol. 50, n 1, p. 68-99.

Gundlach M., Zivnuska S. et Stoner J. (2006). "Understanding the relationship between individualism-collectivism and team performance through an integration of social identity theory and the social relations model", Human Relations, vol. 589, n 12, p. 1603-1632.

Hocquelet M. (2013). «Travailler avec des clients “déviants”. L'expérience des salariés des hypermarchés », Revue française de gestion, vol. 39, n² 234, p. 135-149.

Hogg M.A. et Terry D.J. (2000). "Social identity and self-categorization processes in organizational contexts", Academy of Management Review, vol. 25, n 1, p. 121-140.

Hogg M.A. et Abrams D. (1988). Social Identifications: A social psychology of intergroup relations and group processes, Routledge, Londres.

Jacob M.-R. (2012). Entre travail et organisation : les individus en action dans l'entreprise. Une enquête sur la collaboration entre des salariés et des travailleurs extérieurs dans une grande entreprise, Thèse de doctorat en sciences de gestion, Université Paris-Ouest Nanterre La Défense.

Lallement M. (2007). Le travail. Une sociologie contemporaine, coll. Folio Essais, Éditions Gallimard, Paris.

Lembke S. et Wilson M.G. (1998). "Putting the 'Team' into Teamwork: Alternative Theoretical Contributions for Contemporary Management Practice", Human Relations, vol. $51, \mathrm{n}^{\circ} 7$, p. 927-944.

Musca G. (2006). «Une stratégie de recherche processuelle : l'étude longitudinale de cas enchâssés »,M@n@gement, vol.9,n³,p.153-176. 
164 Revue française de gestion $-\mathrm{N}^{\circ} 240 / 2014$

Rousseau D.M. (1998). "Why workers still identify with organizations", Journal of Organizational Behavior, vol. 19, n 3, p. 217-233.

Tajfel H. (1979). "The achievement of group differentiation”, Differentiation between social groups: Studies in the social psychology of intergroup relations, Tajfel H. (Ed.), Academic Press, Londres, p. 77-98.

Tajfel H. et Turner J.C. (1986). "The social identity theory of intergroup behavior", Psychology of intergroup relations", Worchel S. et Austin W.G. (Eds.), $2^{\text {nd }}$ ed, Nelson-Hall, Chicago, p. 7-24.

Van Der Vegt G.S. et Bunderson J.S. (2005). "Learning and performance in multidisciplinary teams: the importance of collective team identification", Academy of Management Journal, vol. $48, \mathrm{n}^{\circ} 3$, p. 532-547. 